\title{
Near video-rate optical coherence elastography by acceleration with a graphics processing unit
}

\author{
Rodney W. Kirk, Member, IEEE, Brendan F. Kennedy, David D. Sampson, Senior Member, IEEE, \\ Robert A. McLaughlin, Member, IEEE
}

\begin{abstract}
We present a graphics processing unit (GPU)accelerated optical coherence elastography (OCE) system capable of generating strain images (elastograms) of soft tissue at near video-rates. The system implements phase-sensitive compression OCE using a pipeline of GPU kernel functions to enable a highly parallel implementation of OCE processing using the OpenCL framework. Developed on a commercial-grade GPU and desktop computer, the system achieves a processing rate of 21 elastograms per second at an image size of $960 \times 400$ pixels, enabling high-rate visualization during acquisition. The system is demonstrated on both tissue-simulating phantoms and fresh $e x$ vivo mouse muscle. To the best of our knowledge, this is the first implementation of near video-rate $\mathrm{OCE}$ and the fastest reported OCE processing rate, enabling, for the first time, a system capable of computing and displaying OCE elastograms interactively during acquisition. This advance provides new opportunities for medical imaging of soft tissue stiffness using optical methods.
\end{abstract}

Index Terms - optical coherence elastography, OCE, optical coherence tomography, OCT, strain, graphics processing unit, GPU.

\section{INTRODUCTION}

Many diseases alter the mechanical properties of tissue. Optical coherence elastography (OCE) [1-4] is an emerging family of techniques that maps the local mechanical properties of tissue, with the potential to characterize pathological change. Previous work has applied these techniques to a variety of tissues, including cornea [5], cardiac muscle [6] and breast [7]. A range of OCE techniques have been proposed. Each involves the application of a mechanical load to the

The authors wish to thank Lixin Chin and Jessica R. Terrill for their help in performing mouse muscle scanning. This research was supported by grants from the Australian Research Council, the National Health and Medical Research Council (Australia) and Cancer Council Western Australia.

R. W. Kirk, B. F. Kennedy and R. A. McLaughlin are with the Optical+Biomedical Engineering Laboratory, School of Electrical, Electronic and Computer Engineering, University of Western Australia, Crawley, WA 6009, Australia (e-mail: $\quad$ rodney.kirk@uwa.edu.au; brendan.kennedy@uwa.edu.au; robert.mclaughlin@uwa.edu.au).

D. D. Sampson is with the Optical+Biomedical Engineering Laboratory, School of Electrical, Electronic and Computer Engineering, University of Western Australia, Crawley, WA 6009, Australia. He is also with Centre for Microscopy, Characterisation and Analysis, University of Western Australia, Crawley, WA 6009, Australia (david.sampson@uwa.edu.au).

Copyright (c) 2015 IEEE. Personal use of this material is permitted. However, permission to use this material for any other purposes must be obtained from the IEEE by sending a request to pubs-permissions@ieee.org. tissue, measurement of the corresponding tissue displacement using optical coherence tomography (OCT), and estimation of a mechanical property or parameter. In this paper, we focus on a three-dimensional (3D) OCE technique referred to as optical coherence micro-elastography (OCME), in which a compressive load is imparted to an imaging window using an annular piezoelectric transducer [8]. By calculating the phase difference between complex two-dimensional (2D) OCT cross-sectional scans in depth (B-scans) acquired before and after compression, it is possible to measure nano- to microscale displacements in the depth direction at each location in the tissue. Calculating the derivative of displacement with respect to axial depth, we obtain an estimate of the local strain [7], providing an indication of the mechanical properties of the tissue. This is represented graphically as an elastogram.

However, calculation of strain is computationally demanding and time consuming, particularly when applied across the densely sampled 3D data sets acquired in OCME. Recent work has significantly improved the 3D-OCE acquisition speed [9], but computations are still commonly performed offline over a period of minutes to hours. The lack of interactive feedback during imaging is a significant issue in OCME, as scans are subject to imaging artifacts from both optical and mechanical boundaries. Optimal positioning of the sample relative to the imaging window is critical to achieve both an even distribution of the compressive load and good optical coupling, so as to minimize such confounding factors. However, this is impractical when there are large delays between image acquisition and OCE computation. In addition, the time delay in completing the processing chain severely limits the future applicability of OCE techniques to clinical scenarios.

Graphics processing units (GPU) are highly parallel processing devices based on multiple stream processors to enable single instruction, multiple data (SIMD) operations. GPUs can process thousands of data elements simultaneously, supporting highly accelerated processing. The parallel nature of OCT data processing lends itself well to GPU implementation, and previous work has shown significant improvements in computation time for the generation of standard OCT data using GPU processors [10-14]. More recently, several researchers have extended the use of GPUs to more complex processing of OCT data, such as speckle variance [15, 16] and Doppler [17] processing for rapid detection and assessment of blood flow, and polarization- 
sensitive OCT for assessment of birefringence [18].

In this paper, we address the computational challenges inherent in OCE and present the first GPU-based OCE implementation. Our implementation achieves near video-rate computation and display of elastograms enabling, for the first time, the ability to interactively assess the mechanical properties of tissue with OCE.

\section{EXPERIMENT}

Imaging was performed with a spectral-domain OCT system, utilizing a superluminescent diode with central wavelength $835 \mathrm{~nm}$ and a bandwidth of $50 \mathrm{~nm}$, and illuminating the sample with an optical power of $10 \mathrm{~mW}$. The spectrometer incorporated a linescan camera (Model spL4096140k, Basler Sprint, Ahrensburg, Germany) with a line width of 4096 pixels, and scan rate of $140 \mathrm{kHz}$. This was interfaced to a $680 \mathrm{MB} / \mathrm{s}, 12$ bit PCI Express framegrabber (Model PCIe1429, National Instruments, TX, USA) for data capture. For each A-scan, 1792 pixels were acquired, with an exposure time of $2 \mu \mathrm{s}$, and padded out to 2048 pixels during processing. The axial and lateral resolutions were measured to be $7.8 \mu \mathrm{m}$ (in air) and $11 \mu \mathrm{m}$, respectively, with a sensitivity of $102 \mathrm{~dB}$ for an exposure time of $36 \mu \mathrm{s}$. Further details of the system are given in [7].

Experiments to assess the computational performance of the system were performed on both phantoms and ex vivo mouse muscle tissue. A tissue-simulating phantom [19] was fabricated using room-temperature vulcanizing silicone, and contained multiple stiff inclusions (45 times stiffer than the surrounding matrix). Titanium dioxide $\left(\mathrm{TiO}_{2}\right)$ scatterers were added to control the optical properties of the phantom. The same scatterer concentration was used in both the bulk material and inclusions, providing similar optical properties so that delineation of the inclusions relied upon differences in the mechanical properties of the two materials.

Animal studies were approved by The University of Western Australia Animal Ethics Committee. Fresh muscle tissue was obtained from two male, twelve-week-old mice: one wild-type C57 (C57BL/10ScSn) mouse and one dystrophic $m d x$ mouse $\left(\mathrm{C} 57 \mathrm{BL} / 10 \mathrm{ScSn}^{m d x} / m d x\right)$. Gluteus and quadriceps muscles were dissected and kept hydrated in phosphate-buffered saline until imaging (within 1 hour). Samples were mechanically actuated at a rate of $5 \mathrm{~Hz}$ and Bscans were acquired at $10 \mathrm{~Hz}$, one loaded and one unloaded in each cycle of the square wave used to drive the actuator. OCT B-scans and OCME 2D elastograms were computed. The elastograms have dimensions $(x z)$ of $9 \mathrm{~mm} \times 1.2 \mathrm{~mm}$, corresponding to $960 \times 400$ pixels. These were spaced at $1 \mu \mathrm{m}$ intervals and acquired over a $9 \mathrm{~mm}$ field-of-view in the third (y) dimension.

\section{GPU SOFTWARE DESIGN}

OCME processing was implemented in OpenCL 1.1 on an ASUS/Nvidia GeForce GTX 680, (max clock: 1GHz, 1536 cores, 2GB DDR5 memory). An overview of the processing chain is illustrated in the Unified Modeling Language (UML) activity diagram of Fig. 1. Each acquired A-scan is buffered in main memory until a full B-scan has been received, passed to the GPU, and then processed as a series of pipelined operations, referred to as kernels. We have utilized a highly reconfigurable architecture comprising simple, single-purpose kernels that are queued, such that the output of one kernel becomes the input to the next. The approach maximizes code re-usability and facilitates unit testing. During execution, thousands of parallel tasks are created, each applying a kernel to a subset of the data.

It should be noted that the use of multiple, smaller kernels may have some negative impact on performance. GPUs typically have a variety of memory types available, each with different performance characteristics and access speeds. Thus, some of the flexibility afforded by the architecture adopted here could be traded off for still higher processing speed through the use of larger kernels.

In OCME, a densely sampled sequence of B-scans is acquired across the tissue. In our implementation, we used a spacing of $1 \mu \mathrm{m}$ to provide phase correlation between consecutive B-scans. Phase correlation ensures that the phase difference between the same pixels in consecutive scans is proportional to the axial displacement [20]. Processing is applied to pairs of B-scans, with one scan acquired before and the other after mechanical loading. As illustrated in Fig. 1, computation of each B-scan is achieved through kernels performing spectral shaping and background subtraction, followed by zero padding and filtering with a Hann window for noise reduction, prior to computation of an inverse fast Fourier transform. At this stage in the processing, the data was cropped to 400 pixels per A-scans, restricting computations to depths that typically had a higher OCT signal-to-noise ratio.

A phase difference image is calculated using a kernel that subtracts the phase at corresponding locations in the two Bscans [3]. A subsequent kernel applies weighted averaging in the densely sampled $y$ dimension over three pairs of B-scans in order to reduce the phase noise, with each value weighted by the OCT SNR. A 2D implementation of the phase unwrapping algorithm described in [7] is then used to increase the range of measurable displacements. The local strain at each location is calculated as the derivative of tissue displacement with respect to axial depth, computed using the weighted least-squares line of best fit to displacement values within a user-defined axial range around each location $(128 \mu \mathrm{m}$ in the results presented here), where each displacement value is weighted by the SNR of the corresponding OCT signal. Finally, a separate kernel aggregates these estimates of strain into an elastogram, where the intensity of each pixel is linearly related to the local strain. The elastogram is then displayed using OpenGL. Throughout the processing chain, we synchronize data at the output of each kernel, ensuring that all necessary data is available for the next kernel. 


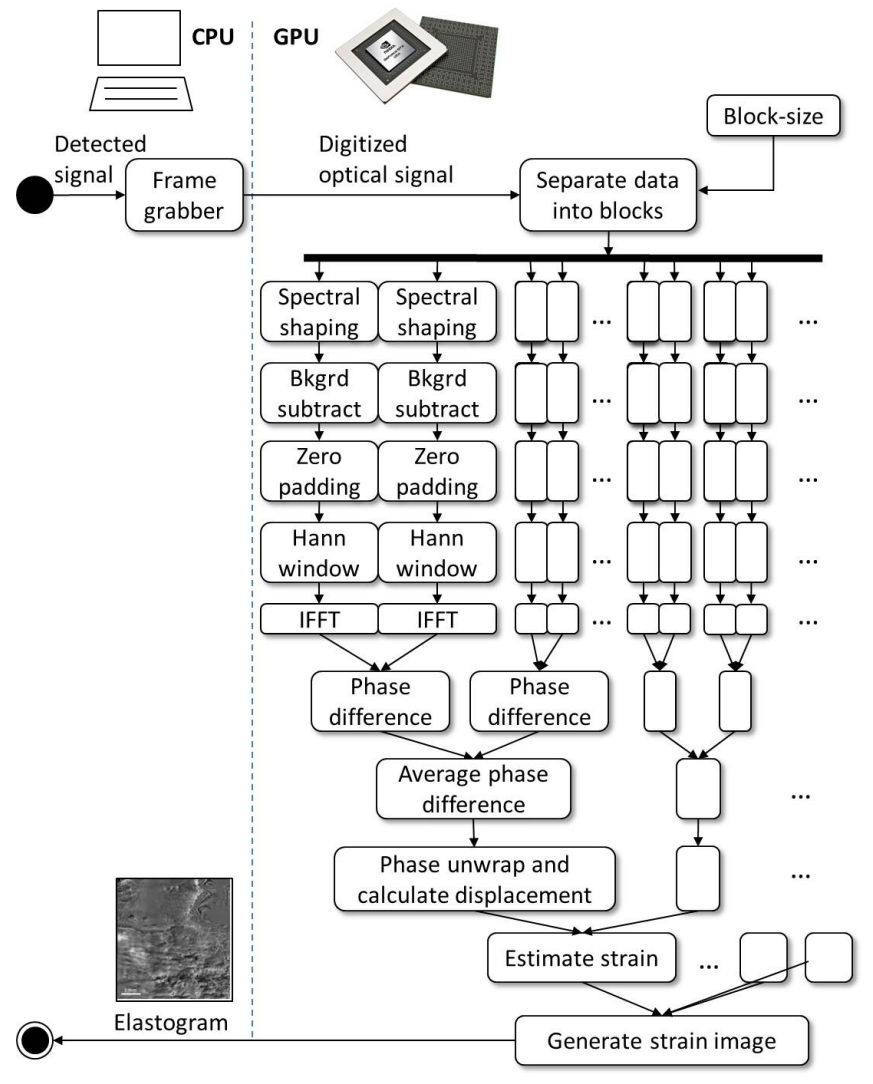

Fig. 1: GPU-accelerated elastography software architecture.
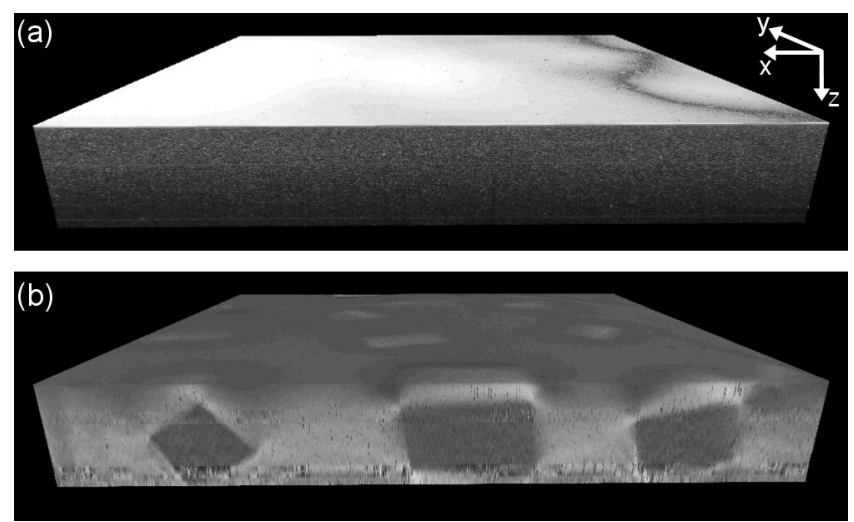

Fig. 2. 3D visualization of a tissue-mimicking phantom. (a) OCT. (b) OCME.

\section{RESUlTS AND Discussion}

Fig. 2(a) and 2(b) show 3D visualizations of the GPUaccelerated 3D-OCT and OCME scans of the tissue-simulating phantom. The visualization data has been cropped so as to display a B-scan intersecting three stiff inclusions. The OCT shows little contrast between the inclusions and bulk materials due to the similarity in their optical properties. In comparison, the OCME shows clear delineation of the stiff inclusions. Animations showing all B-scans from these data sets are given in Media 1, showing OCT (top) and OCME (bottom). The GPU implementation calculated elastograms at a rate of 21 elastograms/s. This is significantly faster than the acquisition rate of 10 pairs of B-scans (loaded and unloaded) per second, which is limited by the rate of mechanical actuation.
A critical parameter in OpenCL GPU-computation optimization is the workgroup size. This is a user-defined, multi-dimensional parameter that indicates the number of work items (i.e., a kernel instance and associated data) executed on each compute unit (i.e., a logical entity that schedules and processes work items). Use of optimal workgroups allows efficient memory coalescing, where multiple global memory accesses are performed in a single transaction. In our implementation, the workgroup size was defined by two values, indicating the $x$ and $y$ extent of a rectangular region across a B-scan. This parameter is specified whenever a kernel is queued for execution on a compute unit and can be individually optimized for each kernel.

To assess the impact of this parameter, we used in-house performance assessment software, which executed the OCME kernel functions with buffered data to avoid the variable latencies associated with data acquisition. The average computation time per elastogram was recorded over a range of workgroup sizes (varying both $x$ and $y$ ) and the results are graphed in Fig. 3(a). Note that each workgroup size may correspond to multiple $x, y$ geometries (i.e., a workgroup of 8 A-scans was parameterized $(x \times y)$ by $1 \times 8 ; 2 \times 4 ; 4 \times 2$; and $8 \times 1$ A-scans). This gives rise to multiple values being graphed for most workgroup sizes, as workgroup shape will also impact performance. While different kernels may have different optimal workgroup sizes, a single workgroup size was chosen for all kernels within each test run during performance testing. We observed monotonically improving performance with increasing workgroup size, with a workgroup size of $(8 \times 8=)$ 64 giving an average computation time (without data acquisition latencies) of $20.9 \mathrm{~ms}$ per elastogram, equivalent to 47 elastograms/s.

Note that the maximum computation rate measured during actual scanning, i.e., 21 elastograms/s, included the latencies associated with data transfer from the framegrabber, task scheduling, and other infrastructure overheads but was not limited by the tissue actuation speed. This is still significantly faster than the image acquisition rate of $10 \mathrm{~Hz}$, which is limited by the mechanism of physical actuation, and thus the GPU implementation lends itself well to processing OCE acquired using faster protocols [9].

The number of work items in a workgroup is limited by the GPU (in our case, the maximum was 1,024). Also, the size of the workgroup in the $x$ and $y$ dimensions must evenly divide into the maximum $x$ and $y$ extents of the data, respectively. Whilst workgroup sizes larger than $8 \times 8$ were evaluated, Fig. 3(a) does not include further sizes as they show relatively little performance improvement. 


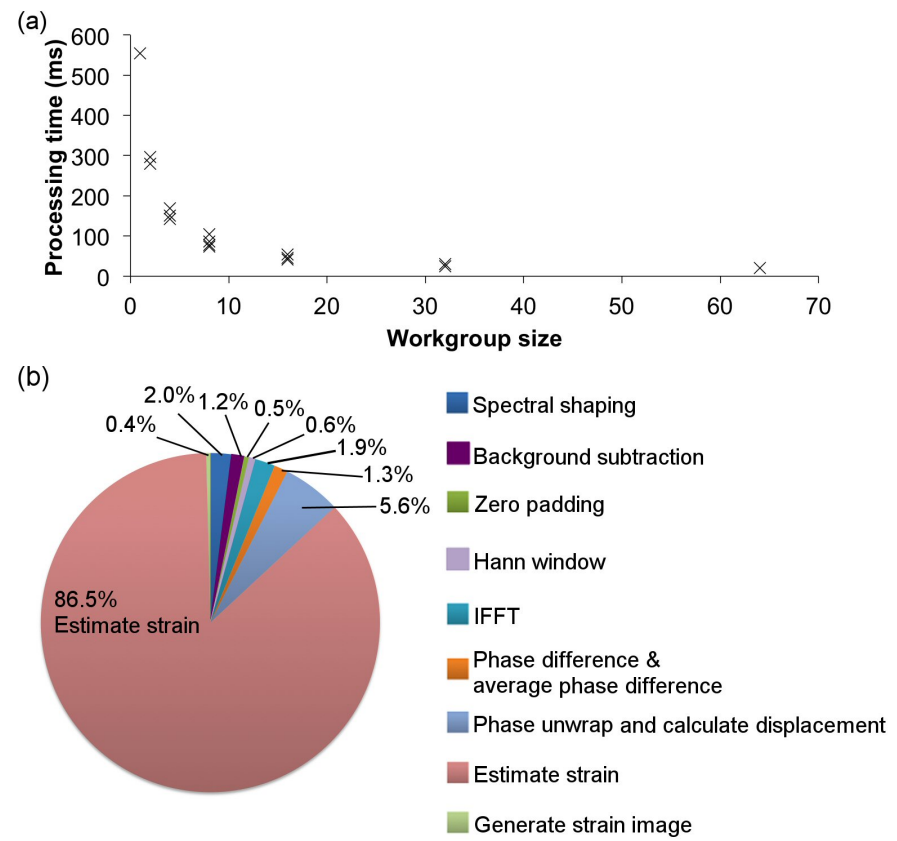

Fig. 3. (a) OCME processing times for a single elastogram, graphed against different workgroup sizes. Multiple geometries are plotted for each size. (b) Relative computation times of each kernel at a workgroup size of 64 .

In addition, the GPU may not be capable of processing all nominated data in parallel, depending on details of the internal architecture and hardware resources. Factors such as the sizes and numbers of internal registers, amount of on-chip memory, capacity of data buses, and number and architecture of internal compute units will dictate the optimal parallel scheduling of simultaneous processes, and the final scheduling of parallel processes is controlled internally by a scheduler on the GPU. Additionally, the optimal block size will vary for different kernels, depending on the specific computations performed. By assigning a kernel-specific optimal block-size for each individual kernel in the computation chain, we were able to achieve an additional $9 \%$ improvement in performance to $19.1 \mathrm{~ms}$ per elastogram (without data acquisition latencies).

Fig. 3(b) shows the relative computation time of each kernel in the processing chain at the workgroup size of $(x \times y) 8 \times 8=$ 64. We note that $86.5 \%$ of the computation time is spent estimating the strain. This is because of the computationally intense operations required to find a line of best fit for a series of axial displacement measurements, where each measurement is weighted by the corresponding OCT SNR. Although the use of such a weighting increases computational complexity, we have found it to be extremely important to minimize the impact of noise on the measured OCE values. In addition, the large range of data over which the line is fitted reduces the use of memory coalescing.

Fig. 4 shows the results of utilizing our GPU-accelerated implementation to generate OCT images and elastograms of tissue, in this case, mouse muscle. Fig. 4(a) and 4(b) show results from a $\mathrm{C} 57$ wild-type mouse. The tissue primarily comprises of muscle fibers, each sheathed by a thin layer of connective tissue. These structures are discernible in the OCT image (Fig. 4a) and clearly delineated as striations in the elastogram (Fig 4b). The muscle fibers exhibit a different mechanical response to the surrounding connective tissue, providing mechanical contrast in elastograms [21]. Using our GPU implementation, we computed elastograms at a rate of 21 elastograms/s, enabling display of elastograms during acquisition. An animation showing a $3 \mathrm{D}$ visualization of the OCME data set is presented in Media 2.

Fig. 4(c) and 4(d) show data acquired from a dystrophic $m d x$ mouse, and demonstrate the ability of GPUimplementation to provide contrast between tissue types. An area of fascia is visible in the center of the bottom half of the images, indicated by the arrow in Fig. 4(d). In the elastogram, this presents as a more homogeneous region than the heavily striated areas of myofibers. Re-orientation of the muscle fibers is apparent in the top right of the image (indicated by the arrow head), presenting as a mottled appearance in the elastogram as described in [21]. Media 3 shows a visualization of this OCME dataset.

\section{CONCLUSION}

We have presented a novel processing architecture that has enabled near-video-rate optical coherence elastography imaging in two dimensions, and supports volume image acquisition. Utilizing GPU-acceleration, we have achieved processing rates of 21 elastograms/s using a commercial-grade GPU and desktop computer. The design utilizes a processing chain of GPU kernels, allowing greater potential for code reuse than is possible with a monolithic design. Results on ex vivo muscle tissue demonstrated the ability of this OCE implementation to provide delineation of structures such as muscle fibers, and differentiation of tissues types such as muscle and fascia. These results demonstrate the system's capability to compute and display elastograms simultaneously with OCE image acquisition, which is a critical step towards the utilization of OCE as a medical imaging modality in in vivo and image-guidance scenarios. 
This article has been accepted for publication in a future issue of this journal, but has not been fully edited. Content may change prior to final publication. Citation information: DOI 10.1109/JLT.2015.2413402, Journal of Lightwave Technology

JLT-17265-2014
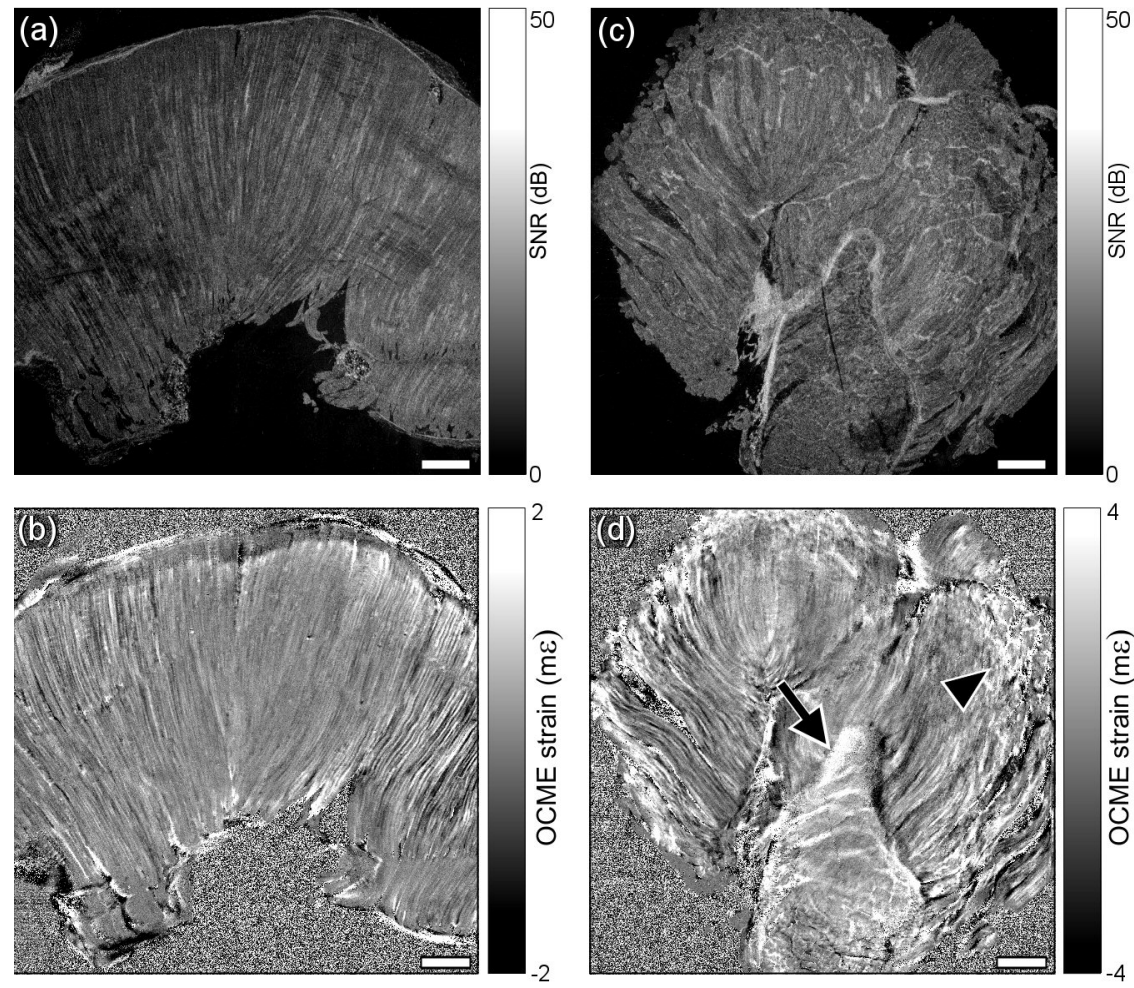

Fig. 4. (a) OCT and (b) OCME of the left gluteus muscle of a C57 wild-type mouse. (c) OCT and (d) OCME of the left quadriceps muscle of an $m d x$ dystrophic mouse. Arrow indicates an area of fascia. Arrowhead indicates muscle tissue orientated perpendicular to the image plane. Scale bar is $1 \mathrm{~mm}$. me, millistrain. See also Media 2 and Media 3.

[9] B. F. Kennedy, F. G. Malheiro, L. Chin, and D. D. Sampson, "Three-dimensional optical coherence elastography by phasesensitive comparison of C-scans," Journal of Biomedical Optics, vol. 19, p. 076006, Jul 2014.

\section{REFERENCES} vol. 14, pp. 11585-11597, Nov 2006. Mar 2011. 2011. Jul 2014.
S. J. Kirkpatrick, R. K. Wang, and D. D. Duncan, "OCT-based elastography for large and small deformations," Optics Express,

C. Sun, B. Standish, and V. X. Yang, "Optical coherence elastography: current status and future applications," Journal of Biomedical Optics, vol. 16, p. 043001, Apr 2011.

B. F. Kennedy, K. M. Kennedy, and D. D. Sampson, "A Review of Optical Coherence Elastography: Fundamentals, Techniques and Prospects," IEEE Journal of Selected Topics in Quantum Electronics, vol. 20, p. 7101217, Mar-Apr 2014.

B. F. Kennedy, X. Liang, S. G. Adie, D. K. Gerstmann, B. C. Quirk, S. A. Boppart, et al., "In vivo three-dimensional optical coherence elastography," Optics Express, vol. 19, pp. 6623-6634,

D. Alonso-Caneiro, K. Karnowski, B. J. Kaluzny, A. Kowalczyk, and M. Wojtkowski, "Assessment of corneal dynamics with highspeed swept source optical coherence tomography combined with an air puff system," Optics Express, vol. 19, pp. 14188-14199, Jul

S. Wang, A. L. Lopez, Y. Morikawa, G. Tao, J. Li, I. V. Larina, et al., "Noncontact quantitative biomechanical characterization of cardiac muscle using shear wave imaging optical coherence tomography," Biomedical Optics Express, vol. 5, pp. 1980-1992,

B. F. Kennedy, R. A. McLaughlin, K. M. Kennedy, L. Chin, A. Curatolo, A. Tien, et al., "Optical coherence micro-elastography: mechanical-contrast imaging of tissue microstructure," Biomedical Optics Express, vol. 5, pp. 2113-2124, Jul 2014.

B. F. Kennedy, T. R. Hillman, R. A. McLaughlin, B. C. Quirk, and D. D. Sampson, "In vivo dynamic optical coherence elastography using a ring actuator," Optics Express, vol. 17, pp. 21762-21772, Nov 2009.
[10] Y. Watanabe and T. Itagaki, "Real-time display on Fourier domain optical coherence tomography system using a graphics processing unit," Journal of Biomedical Optics, vol. 14, p. 060506, Nov-Dec 2009.

[11] Y. Watanabe, S. Maeno, K. Aoshima, H. Hasegawa, and H. Koseki, "Real-time processing for full-range Fourier-domain optical-coherence tomography with zero-filling interpolation using multiple graphic processing units," Applied Optics, vol. 49, pp. 4756-4762, Sep 2010.

[12] S. Van der Jeught, A. Bradu, and A. G. Podoleanu, "Real-time resampling in Fourier domain optical coherence tomography using a graphics processing unit," Journal of Biomedical Optics, vol. 15, p. 030511, May-Jun 2010.

[13] K. Zhang and J. U. Kang, "Real-time intraoperative 4D full-range FD-OCT based on the dual graphics processing units architecture for microsurgery guidance," Biomedical Optics Express, vol. 2, pp. 764-770, 2011.

[14] J. Rasakanthan, K. Sugden, and P. H. Tomlins, "Processing and rendering of Fourier domain optical coherence tomography images at a line rate over $524 \mathrm{kHz}$ using a graphics processing unit," Journal of Biomedical Optics, vol. 16, p. 020505, Feb 2011.

[15] K. K. C. Lee, A. Mariampillai, J. X. Z. Yu, D. W. Cadotte, B. C. Wilson, B. A. Standish, et al., "Real-time speckle variance sweptsource optical coherence tomography using a graphics processing unit," Biomedical Optics Express, vol. 3, pp. 1557-1564, Jul 2012.

[16] J. Xu, K. Wong, Y. Jian, and M. V. Sarunic, "Real-time acquisition and display of flow contrast using speckle variance optical coherence tomography in a graphics processing unit," Journal of Biomedical Optics, vol. 19, p. 026001, Feb 2014.

[17] M. Sylwestrzak, D. Szlag, M. Szkulmowski, I. Gorczynska, D. Bukowska, M. Wojtkowski, et al., "Four-dimensional structural and Doppler optical coherence tomography imaging on graphics processing units," Journal of Biomedical Optics, vol. 17, p. 100502, Oct 2012. 
This article has been accepted for publication in a future issue of this journal, but has not been fully edited. Content may change prior to final publication. Citation information: DOI 10.1109/JLT.2015.2413402, Journal of Lightwave Technology

JLT-17265-2014

[18] Y. Wang, C. M. Oh, M. C. Oliveira, M. S. Islam, A. Ortega, and B. H. Park, "GPU accelerated real-time multi-functional spectraldomain optical coherence tomography system at $1300 \mathrm{~nm}, "$ Optics Express, vol. 20, pp. 14797-14813, Jul 2012.

[19] G. Lamouche, B. F. Kennedy, K. M. Kennedy, C.-E. Bisaillon, A. Curatolo, G. Campbell, et al., "Review of tissue simulating phantoms with controllable optical, mechanical and structural properties for use in optical coherence tomography," Biomedical Optics Express, vol. 3, pp. 1381-1398, Jun 2012.

[20] L. Chin, A. Curatolo, B. F. Kennedy, B. J. Doyle, P. R. T. Munro, R. A. McLaughlin, et al., "Analysis of image formation in optical coherence elastography using a multiphysics approach," Biomedical Optics Express, vol. 5, pp. 2913-2930, Sep 2014.

[21] L. Chin, B. F. Kennedy, K. M. Kennedy, P. Wijesinghe, G. J. Pinniger, J. R. Terrill, et al., "Three-dimensional optical coherence micro-elastography of skeletal muscle tissue," Biomedical Optics Express, vol. 5, pp. 3090-3102, Sep 2014. 